\title{
Optimized syntheses of iodylarenes from iodoarenes, with sodium periodate as the oxidant. Part II
}

\author{
Lukasz Kraszkiewicz and Lech Skulski* \\ Chair and Laboratory of Organic Chemistry, Faculty of Pharmacy, Medical University, \\ 1 Banacha Street, PL 02-097 Warsaw, Poland \\ E-mail: Iskulski@farm.amwaw.edu.pl
}

Dedicated to Professor Anastasios Varvoglis on his $65^{\text {th }}$ birthday

(received 4 Feb 03; accepted 28 Mar 03; published on the web 06 May 03)

\begin{abstract}
An improved method is reported for optimized preparations of iodylarenes, $\mathrm{ArIO}_{2}$, from iodoarenes, ArI, using $\mathrm{NaIO}_{4}$ as the oxidant dissolved in boiling $30 \%$ (v:v) aq. $\mathrm{CH}_{3} \mathrm{COOH}$ solutions. The former reaction times, reported in Part I, ${ }^{1}$ were shortened from 8-16 hours to 3-6 hours, with preserving the same good yields and high purities of the crude final products, $\mathrm{ArIO}_{2}$. A considerably improved method of preparing ,2-iodosylbenzoic acid” is also reported in this paper. See also our Warning submitted in Experimental.
\end{abstract}

Keywords: Iodylarenes, 2-iodosylbenzoic acid, iodoarenes, sodium periodate, oxidation

\section{Introduction}

During the past 20 years, iodylarenes, $\mathrm{ArIO}_{2}$, the aromatic compounds of iodine( $V$ ), have widely been used in organic synthesis as mild and very selective oxidants. The most important iodylarenes are: iodylbenzene, 4-(t-butyl)iodylbenzene, "2-iodylbenzoic acid" [i. e. 1-hydroxy1,2-benziodoxol-3-(1H)-one 1-oxide, IBX], and its derivative named the Dess-Martin reagent [1,1,1-triacetoxy-1,2-benziodoxol-3-(1H)-one.$^{2}$

It is known that large quantities of some organophosphorus poisons, viz. Sarin, Soman, Tabun, VX, or Russian-VX are still stockpiled in a number of countries around the world, and their destruction requires the use of environmentally friendly processes. It has been discovered that the conjugate bases of 2-iodosyl- and 2-iodylbenzoic acids, i.e. 2-iodosyl- and 2iodylbenzoates, may successfully be used for the degradation of organophosphorus substrates, including those mentioned above. ${ }^{3}$ This is why we believe that our novel easy and fairly effective syntheses of 2-iodosyl- or 2-iodylbenzoic acids may be of a practical interest. 
In Part $\mathrm{I}^{1}{ }^{\text {we }}$ weported a new method for preparing iodylarenes from iodoarenes, in boiling and vigorously stirred neutral aqueous sodium periodate solutions. These reactions proceeded within 8-16 hours and gave $\mathrm{ArIO}_{2}$ in 58-91\% crude yields, having 97-99\% purities established iodometrically. ${ }^{4}$ We have recently tried, in vain, to accelerate these reactions with microwave irradiation.

The aim of Part II has been to notably shorten the former prolonged reaction times, ${ }^{1}$ but with preserving the former good yields and high purities of the crude final products, $\mathrm{ArIO}_{2}$ - which next may be used as such, without purification, in subsequent reactions. We have also attempted to considerably improve our former synthesis ${ }^{1}$ of „2-iodosylbenzoic acid”.

\section{Results and Discussion}

Recently, in our laboratory we have devised an improved method for optimized preparations of $\mathrm{ArIO}_{2}$ from ArI. We observed that when the aforementioned boiling reaction mixtures were acidified with dilute $\mathrm{H}_{2} \mathrm{SO}_{4}$, the yellowish iodosylarenes, ArIO, were the main products of the reactions. When we used varied boiling aqueous $\mathrm{CH}_{3} \mathrm{COOH}$ solutions, the initially precipitated out yellowish ArIO, after 0.5-1.5 h were transformed into colorless $\mathrm{ArIO}_{2}$. According to these observations, we previously suggested ${ }^{1}$ that the reactions underwent in two steps: ArI are oxidized to only ArIO, and the latter ones are readily thermally disproportionated to give ArI and $\mathrm{ArIO}_{2}$. We established experimentally that the optimum concentration of aqueous $\mathrm{CH}_{3} \mathrm{COOH}$ solutions for preparing $\mathrm{ArIO}_{2}$ was just 30\% (v:v). Using this binary acidic solvent, we considerably shortened the reaction times to $3-6$ hours and, in some cases, we also increased the crude yields. Iodometric titrations ${ }^{4}$ showed $96-99 \%$ purities of the crude iodylarenes obtained.

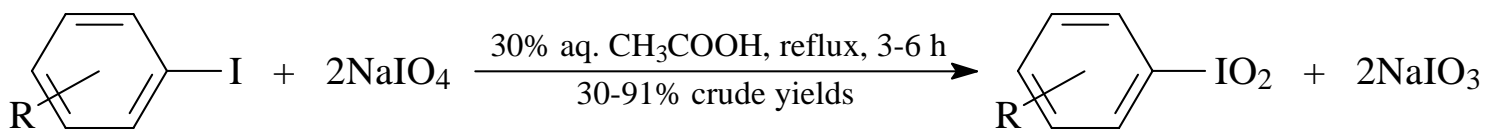

\section{Scheme 1}

$\mathrm{R}$ - see Table 1.

Table 1. The reaction times, crude yields, and melting points (uncorrected) for the recrystallized $\mathrm{ArIO}_{2}$ (Scheme 1)

\begin{tabular}{ccccc}
\hline $\mathrm{R}$ & Time $[\mathrm{h}]^{\mathrm{a})}$ & Crude yield [\%] & $\mathrm{Mp}\left[{ }^{\circ} \mathrm{C}\right]$ & Lit. mp $\left[{ }^{\circ} \mathrm{C}\right]$ \\
\hline $\mathrm{H}$ & 4 & 76 & 235 (expl.) & $235(\operatorname{expl} .)^{1}$ \\
& 6 & 84 & &
\end{tabular}




\begin{tabular}{|c|c|c|c|c|}
\hline $2-\mathrm{CH}_{3}$ & 4 & 70 & 206-207 & $208^{1}$ \\
\hline $3-\mathrm{CH}_{3}$ & $3(+1)$ & 75 & 218 (expl.) & 220 (expl.) $)^{1}$ \\
\hline $4-\mathrm{CH}_{3}$ & $3(+1)$ & 84 & 227 (expl.) & 229 (dec. $)^{1}$ \\
\hline $2-\mathrm{Cl}$ & $5(+1)$ & 40 & $205-206$ & 203 (expl.) \\
\hline 3-Cl & $3(+1)$ & 75 & 231 (expl.) & 233 (expl.) \\
\hline 4-Cl & $3(+1)$ & 87 & 240 (expl.) & $\begin{array}{l}232 \text { (expl.) } \\
243 \text { (expl.) }\end{array}$ \\
\hline $2-\mathrm{NO}_{2}$ & $3(+1)$ & 54 & 212 (expl.) & 210 (expl. $^{5}$ \\
\hline $3-\mathrm{NO}_{2}$ & $3(+1.5)$ & 84 & 215 (expl.) & 214 (dec. $)^{1}$ \\
\hline $4-\mathrm{NO}_{2}$ & $3(+1)$ & 58 & 214 (expl.) & 215 (dec.) $)^{1}$ \\
\hline $3-F$ & 4 & 91 & $226-227$ & $226^{5}$ \\
\hline $4-F$ & $2(+1)$ & 87 & 245 (expl.) & $248^{1}$ \\
\hline $4-\mathrm{Br}$ & $3(+1.5)$ & 81 & 236 & $\begin{array}{l}231 \text { (expl.) } \\
240 \text { (expl.) }\end{array}$ \\
\hline $2,4-\left(\mathrm{CH}_{3}\right)_{2}$ & 4 & 54 & 194 (expl.) & $195^{5}$ \\
\hline $2,4-\mathrm{Cl}_{2}$ & $3(+1)$ & 30 & 223 (expl.) & $225^{5}$ \\
\hline
\end{tabular}

Note: Satisfactory microanalyses, $\mathrm{I} \% \pm 0.3$, were obtained for the purified $\mathrm{ArIO}_{2}$; their IR spectra were the very same as those reported in Part I. ${ }^{1}$

${ }^{\mathrm{a}}$ The reaction times after adding an additional volume of boiling water $(20 \mathrm{~mL})$ are given in parentheses.

2-Iodobenzoic acid, after 4 hours of its boiling with a solution of $\mathrm{NaIO}_{4}$ in $30 \%$ (v:v) aq. $\mathrm{CH}_{3} \mathrm{COOH}$, gave the nearly pure (98-99\%), colorless ,2-iodosylbenzoic acid” [its stable cyclic tautomeric form is named: 1-hydroxy-1,2-benziodoxol-3(1H)-one] in 91-93\% crude yields; for more details see Experimental, and cf. Part I. ${ }^{1}$ However, 3- and 4-iodobenzoic acids, reacted similarly as 2-iodobenzoic acid, gave only mixtures of the corresponding iodyl- and iodosylbenzoic acids; such mixtures, analyzed iodometrically, ${ }^{4}$ may also be used for the degradation of organophosphorus substrates. See Part $\mathrm{I}^{1}$ for the effective methods of preparing the crude 2-, 3- and 4-iodylbenzoic acids, all having 98-99\% purities established iodometrically. ${ }^{4}$

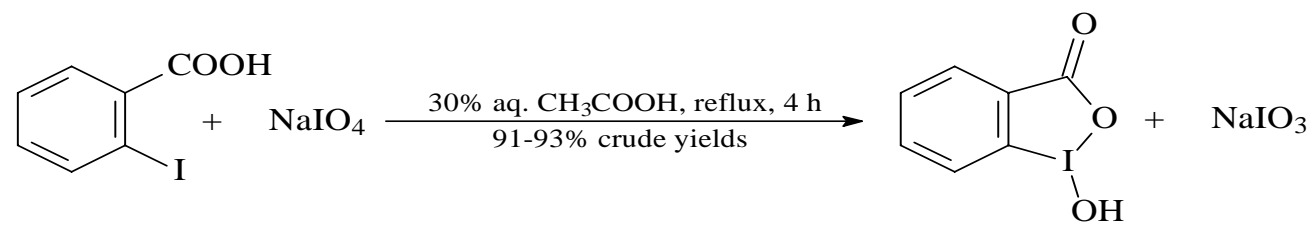

\section{Scheme 2}

\section{Conclusions}

From the above experiments it is seen that our preliminary aims (vide supra) were successfully attained in the present Part II. It should be emphasized that all our crude final products, $\mathrm{ArIO}_{2}$ 
and 2-OIC ${ }_{6} \mathrm{H}_{4} \mathrm{COOH}$, had always high purities (96-99\%), hence they may be used as such, without purification, in all possible subsequent reactions. Our Warning (see Experimental) evidences that even recrystallizations of $\mathrm{ArIO}_{2}$ from boiling water may sometimes be hazardous, particularly when they are scaled up; they are also always connected with unavoidable crystallization losses.

\section{Experimental Section}

General Procedures. All the reagents and solvents were commercial (Aldrich, Fluka), and were used without purification. Chemical structures of the purified compounds in Table 1 were confirmed by satisfactory microanalyses obtained at the Institute of Organic Chemistry, the Polish Academy of Sciences, Warsaw, where also the IR spectra were recorded.

We have observed that melting/decomposition points of many $\mathrm{ArIO}_{2}$ are rather uncertain, depending not only upon the purity of crude or purified products prepared, but also upon the rate of their heating during the melting point determinations; this is why their melting points reported in the literature ${ }^{1,5,6}$ often differ significantly. The melting points of $\mathrm{ArIO}_{2}$ (Table 1) were uncorrected, and often accompanied by explosion. They were measured as follows: after an approximate m.p. had been taken in an open capillary tube, a new sample was introduced about $10{ }^{\circ} \mathrm{C}$ below this point, and the temperature was raised at a rate of $10{ }^{\circ} \mathrm{C} \mathrm{min}{ }^{-1}$. This was repeated unless consistent results were attained.

\section{Optimized procedures for preparing iodylarenes from iodoarenes}

$\mathrm{NaIO}_{4}$ (4.70 g, $22 \mathrm{mmol}$, 10\% excess) was suspended in 30\% (v:v) aq. AcOH (20 mL). An appropriate iodoarene $(10 \mathrm{mmol})$ was added (for steam-volatile ArI, a few drops of toluene were also added; see Part $\mathrm{I}^{1}$ for the explanation). The mixtures were vigorously stirred and refluxed for 2-6 h. In such cases, when the mixtures were still yellowish after this time, $20 \mathrm{~mL}$ of boiling water was added, and the resulting mixtures were further stirred and refluxed for 1-1.5 h (see Table 1). Next, the temperature was lowered to r. t. After $1 \mathrm{~h}$, the crude products were collected by filtration, washed on the filter with ice water $(3 \times 10 \mathrm{~mL})$ and acetone $(3 \times 10 \mathrm{~mL})$, and airdried in the dark. As previously, ${ }^{1}$ small samples of the crude $\mathrm{ArIO}_{2}$ were recrystallized, with effective stirring, from boiling water to obtain the analytical specimens.

Optimized procedure for preparing "2-iodosylbenzoic acid", i.e. 1-Hydroxy-1,2-benziodoxol3(1H)-one. $\mathrm{NaIO}_{4}$ (2.25 g, $10.5 \mathrm{mmol}$; 5\% excess) and 2-iodobenzoic acid (2.48 g, $10 \mathrm{mmol}$ ) were suspended in $30 \%(\mathrm{v}: \mathrm{v})$ aq. AcOH $(15 \mathrm{~mL})$. The mixture was vigorously stirred and refluxed for $4 \mathrm{~h}$. Next, the reaction mixture was diluted with cold water (50 mL) and cooled to $\mathrm{r}$. t. After $1 \mathrm{~h}$, the crude product was collected by filtration, washed on the filter with ice water $(3 \mathrm{x}$ $10 \mathrm{~mL}$ ) and acetone (3 x $10 \mathrm{~mL}$ ), and air-dried in the dark to give 91-93\% yields. The colorless crude products thus obtained repeatedly were analyzed iodometrically ${ }^{4}$ to show $98-99 \%$ purities, 
m.p. $254{ }^{\circ} \mathrm{C}$ (dec.); for the purified compound, m.p. $256{ }^{\circ} \mathrm{C}$ (dec.) (recrystallized, with stirring, from boiling water); m.ps. $234{ }^{\circ} \mathrm{C}$ (dec.) and $260{ }^{\circ} \mathrm{C}$ (dec.) were reported. ${ }^{1,5,6}$

For $\mathrm{C}_{7} \mathrm{H}_{5} \mathrm{IO}_{3}$ calcd $\mathrm{C}, 31.85 ; \mathrm{H}, 1.91 ; \mathrm{I}, 48.06 \%$; for the purified compound found: $\mathrm{C}, 31.8$; H, 2.0; I, 47.9\%; for the crude product found: C, 32.0; H, 2.1; I, 47.9\%.

\section{Warning}

Explosion during the recrystallization of 3-iodyltoluene from boiling water. Dry iodylarenes, $\mathrm{ArIO}_{2}$, are generally hazardous compounds, which may explode upon impact, by scratching them with a spatula, on heating under confinement, etc. ${ }^{2}$ They should always be handled with care, even during their preparations by oxidative reactions, or when they are purified by recrystallization from boiling water (vide infra).

In the course of our preparations of numerous $\mathrm{ArIO}_{2}$ from the corresponding ArI, we recrystallized crude $\mathrm{ArIO}_{2}$ from boiling water, always with vigorous stirring, to obtain the analytical specimens. But for a first time, a violent explosion occured during the recrystalization of ca. $2 \mathrm{~g}$ of 3-iodyltoluene from boiling water. The blast of the explosion was such that fragments of the glassware scratched a transparent security shield made of Plexiglas, while a nest-shaped electric heater coated with asbestos was demolished. No one in the laboratory suffered injuries, due to the presence of the shield.

After the accident, we scrutinized a possible cause of the explosion. We came to the conclusion that it had probably been due to ineffective stirring the boiling biphasic system. It is known ${ }^{2}$ that solid $\mathrm{ArIO}_{2}$ has a polymeric nature, which makes it insoluble in most ordinary solvents, except water. During preparation and recrystallization, the densely-packed heavy crystals of $\mathrm{ArIO}_{2}$ tend to settle down and to adhere to the walls of round-bottomed flasks, if the stirring is ineffective. Because the external electric heating of the flask was applied, hence local overheating the solid $\mathrm{ArIO}_{2}$, adhering tightly to hot walls of the flask, resulted in the explosion. Hence, very effective stirrers, e.g. such as that of the Hershberg-type, ${ }^{7}$ should be used, which scrape out and stir the solids clinging obstinately to the walls of the used vessels. Also during the preparations of $\mathrm{ArIO}_{2}$ from ArI, a very efficient stirring should be applied to sustain the heavy $\mathrm{ArIO}_{2}$ crystals in the form of a suspension in the bulk of a boiled liquid phase heated externally.

Our preliminary results were presented at the Sixth Electronic Conference on Synthetic Organic Chemistry (ECSOC-6, http://www.mdpi.net/ecsoc-6/), September 1-30, 2002 (paper A014).

\section{References and Notes}

1. Kazmierczak, P.; Skulski, L.; Kraszkiewicz, L. Molecules 2001, 6, 881. Avail. at URL: http://www.mdpi.org/molecules/papers/61100881.pdf 
2. (a) Varvoglis, A. The Organic Chemistry of Polycoordinated Iodine, VCH: Weinheim, 1992; (b) Stang, P. J.; Zhdankin, V. V. Chem. Rev. 1996, 96, 1123; (c) Varvoglis, A. Hypervalent Iodine in Organic Synthesis, Academic Press: San Diego, 1997; (d) Zhdankin, V. V.; Stang, P. J. Chem. Rev. 2002, 102, 2523.

3. Morales-Rijas, H.; Moss, R. A. Chem. Rev. 2002, 102, 2497.

4. Lucas, H. J.; Kennedy, E. R.; Formo, M. W. Org. Synth. 1942, 22, 70.

5. Beringer, F. M.; Gindler, E. M. Iodine Abstr. Rev. 1956, 3, 1.

6. Aldrich Handbook of Fine Chemicals and Laboratory Equipment 2003-2004, p 1075; a m.p. of $230{ }^{\circ} \mathrm{C}$ (dec.) is reported for 97\% 2-iodosobenzoic acid, with a note: LIGHTSENSITIVE.

7. Vogel, A. I. A Text-book of Practical Organic Chemistry Including Qualitative Organic Analysis, Longmans and Green: London, 1948; p 65. 